\title{
Impact des rejets d'assainissement permanents ou transitoires sur la qualité des eaux de baignade
}

\author{
F. BLANCHET', E. SOYEUX'2, J.-C. DEUTSCH ${ }^{3}$, Y.H. DE ROECK ${ }^{4}$
}

Mots-clés : qualité des eaux de baignade, rejets d'assainissement, modélisation courantologique, maîtrise opérationnelle des risques.

\section{Introduction}

La qualité des eaux de baignade est à la croisée de trois enjeux :

- un enjeu sanitaire, lié à l'exposition éventuelle des baigneurs aux virus ou aux germes ;

- un enjeu environnemental ; ce sont les pollutions à l'interface du milieu terrestre et marin qui peuvent être à l'origine d'une dégradation chronique ou transitoire de la qualité des eaux de baignade ;

- un enjeu économique, car elle constitue un déterminant pour la fréquentation touristique des stations balnéaires.

Cet article se propose de dresser un panorama de la question des impacts des rejets d'assainissement sur la qualité des eaux de baignade, à travers :

- une description du contexte scientifique, technique et réglementaire ;

- une typologie des différentes sources de rejets ;

- les voies d'amélioration possibles et l'importance de la courantologie côtière dans la définition des solutions techniques;

1 Veolia Eau - Direction technique. 1, rue Giovanni Battista Pirelli 94410 Saint-Maurice France.

2 Veolia Environnement - Direction de la Recherche, du Développement et de la Technologie - 38, avenue Kléber, 75116 Paris France.

3 Centre d'enseignement et de recherche sur l'eau, la ville et l'environnement - 6-8, av. Blaise Pascal - Cité Descartes, Champs-sur-Marne, 77455 Marne-la-Vallée Cedex 2 France.

4 Ifremer - Département "Dynamiques de l'environnement côtier" - BP 70, 29280 Plouzané France.
- enfin, nous aborderons l'articulation entre la « gestion active» des risques et la surveillance des systèmes d'assainissement en zone littorale.

\section{Contexte scientifique, technique et réglementaire}

\subsection{Contexte scientifique et technique}

À travers l'analyse des déversements côtiers sur la qualité des eaux de baignade et des diverses voies pour en limiter les impacts, un certain nombre d'aspects techniques et scientifiques sont abordés. Cependant, cette approche explicite recouvre en fait des problématiques implicites qui sont au moins aussi importantes pour comprendre comment il est possible d'améliorer la qualité des eaux de baignade en zone littorale. Avant de rentrer dans le vif du sujet, il ne semble donc pas inutile d'examiner ces problématiques qui sont au moins au nombre de trois :

- la prise de conscience de la pollution des rejets urbains de temps de pluie ;

- la gestion intégrée de l'eau ;

- l'acquisition des connaissances entre les domaines de l'opérationnel et celui de la recherche.

Les eaux pluviales urbaines, et plus largement les rejets urbains de temps de pluie, sont un des sujets dont il sera question plus largement par la suite. La connaissance de leur pollution a été un véritable sujet à éclipses pendant tout le xx siècle. De nombreux articles indiquent que l'on a une conscience relativement bonne vers la fin du XIX ${ }^{e}$ siècle. Dans [BECHMANN, 1899], on indique sans précautions 
oratoires superflues : "D'après M. le Professeur Cornil, les eaux des rues contiennent une multitude de germes, parmi lesquels ceux de maladies infectieuses comme le tétanos, la phtisie, la pneumonie, la septicémie, etc.

Les recherches, peu nombreuses il est vrai, faites sur le même objet à l'étranger, ont conduit exactement aux mêmes constatations. "

[GOUBERT, 1986] indique que vers la fin du $\mathrm{XIX}^{\mathrm{e}}$ siècle, la méfiance grandit à l'endroit des citernes d'eaux pluviales et donne plusieurs récits où les citernes ont été la cause d'une maladie.

À partir du début du xx siècle toutes les références à ce problème disparaissent. La focalisation sur le traitement biologique des eaux usées et par voie de conséquence, la mise en œuvre des réseaux séparatifs, en est probablement une des causes.

Il faudra attendre la fin des années 1960 pour que l'on recommence à se préoccuper de la pollution des eaux pluviales urbaines. Dans la foulée, quelques articles apparaissent dans la littérature pour formuler des indications sur la contamination bactériologique de ces eaux et sur leur impact potentiel dans les eaux de surface, mais ils sont encore rares. Vraisemblablement, l'idée qu'il faut considérer l'ensemble des rejets et pas simplement les rejets d'eaux usées, considérés a priori comme les plus importants, a du mal à faire son chemin.

La directive cadre européenne sur la gestion de l'eau a porté au rang de principe une idée qui avait commencé à être appliquée en France : le bon niveau territorial pour la gestion de l'eau est celui du bassinversant. À ce niveau, l'ensemble des apports, des rejets, des impacts et des utilisations doivent être considérés ; c'est ce qu'on appelle la « gestion intégrée ». L'application à notre sujet conduit à prendre en compte tous les rejets. Une spécificité, qui est aussi une complication existe cependant dans le cas de la frange littorale : ce n'est pas seulement le ou les bassins-versants dont l'exutoire aboutit à la zone côtière à laquelle on s'intéresse qu'il faut étudier. Il est aussi indispensable de quantifier les rejets qui aboutissent sur la même zone par l'effet des courants côtiers.

La multiplication des acteurs sociaux impliqués due à l'extension du territoire hydraulique et hydrologique concerné ne facilite alors pas la mise en œuvre de ou des solutions nécessaires, que celles-ci soient techniques ou institutionnelles.

Comme la grande majorité des questions d'environnement, la solution en vue de l'amélioration de la qualité des eaux de baignade fait appel aussi bien à des connaissances locales qu'à des connaissances générales. On l'a vu plus haut, peu de recherches ont été entreprises dans ce domaine. Même si on connaît aujourd'hui les procédures de mesure de la pollution bactériologique, il est difficile de se faire une idée sur la variation de cette pollution dans le cadre des rejets transitoires. De même, sa rémanence dans le milieu marin en fonction des conditions climatologiques, ainsi que l'impact sanitaire qu'elle est susceptible d'avoir, constituent des sujets pour lesquels des retours d'expériences sont susceptibles d'améliorer les connaissances. Il est essentiel d'avancer sur ces points qui sont des « passages obligés » pour nourrir la prise de décision concernant les solutions à mettre en ouvre.

\section{2. Évolutions récentes du contexte réglementaire}

La nouvelle directive européenne de gestion de la qualité des eaux de baignade (directive 2006/7/CE du 15 février 2006) introduit différentes évolutions majeures, notamment :

- les règles de classement des zones de baignade ;

- une volonté accrue d'information des usagers des plages;

- le principe des « plans de gestion des risques » liés aux dégradations ponctuelles de la qualité des eaux de baignade qui pourront conduire, le cas échéant, à des mesures d'interdiction ponctuelles de la baignade, dispositif qui sous-entend un niveau minimal de « maîtrise opérationnelle du risque » de dégradation de la qualité de l'eau.

La directive européenne préconise ou suggère certains outils techniques, au titre d'obligation de moyens :

- profil de vulnérabilité des plages : inventaire des sources de pollution et de leur criticité ;

- surveillance des sources de pollution reconnues comme les plus critiques (instrumentation permanente, pluviométrie, télégestion, surveillance régulière, etc.) ; 
- évaluation régulière (quotidienne) du risque de dégradation de la qualité des eaux de baignades compte tenu des informations de contexte disponibles à travers le système de surveillance.

De plus, pour les zones de baignade de qualité « insuffisante », des mesures adéquates d'éradication et/ou de diminution des sources de pollution devront être mises en œuvre.

Pour les sources de pollution chroniques ou transitoires émises par les systèmes d'assainissement, cette nouvelle directive présente de fortes cohérences avec d'autres textes réglementaires :

- la directive ERU de 1991 et le principe d'autosurveillance des systèmes d'assainissement (au moins pour les plus de $2000 \mathrm{EH}$ ), puisque la surveillance des sources de pollution prévue aux plans de gestion pourront parfaitement intégrer les équipements d'autosurveillance en place ;

- la DCE, puisque sur les zones de baignade jugées insuffisantes, l'éradication ou la diminution des sources de pollution à moyen terme vise à rétablir un « bon état écologique » compatible avec les usages « en aval».

\section{Typologie des rejets d'assainissement}

Pour établir un profil de vulnérabilité d'une plage, point de départ de la réflexion sur la qualité des eaux de baignade, il convient de distinguer et de hiérarchiser l'origine des flux de pollution et leur intensité dans le temps. De cette première réflexion découlent les moyens à mettre en ouvre pour juguler ces flux ou maîtriser leurs impacts.

\subsection{Origine et nature des rejets}

Les origines des flux de pollution les plus évidentes sont celles situées à proximité immédiate de la plage considérée : les rivières côtières et les systèmes d'assainissement. Cependant, une plage peut également être sous l'influence du flux de pollution provenant de zones plus lointaines, par exemple lorsque se combinent rejets significatifs et courants côtiers défavorables. Sur ces rejets provenant de territoires voisins, soit situés sur le littoral soit dispersés sur le bassin-versant des rivières côtières, les moyens d'action pour la collectivité gestionnaire de la plage sont plus limités que sur son système d'assainissement.
Quelle que soit leur origine, on peut distinguer trois natures de rejets : chronique, accidentelle et transitoire.

Pour les rejets chroniques, citons les rivières côtières, les rejets d'usines de dépollution des eaux usées et les réseaux d'eaux pluviales transportant des eaux usées. Les rejets urbains de temps de pluie peuvent quant à eux être qualifiés de rejets transitoires avec comme principales manifestations : les déversoirs d'orage de réseaux unitaires, les by-pass d'usine de dépollution des eaux usées et les réseaux pluviaux. Des rejets, que nous qualifierons d'accidentels, peuvent également produire un effet considérable, en particulier lorsqu'ils sont situés à proximité immédiate d'une plage. Certains sont directement liés au réseau d'assainissement : panne d'un poste de relèvement d'eaux usées engendrant un débordement (incident potentiellement maîtrisable par l'usage de la télésurveillance) ou les obstructions de réseau d'eaux usées donnant lieu à un passage au pluvial (par l'intermédiaire d'un regard mixte ou d'un branchement). D'autres rejets ont des causes plus insidieuses: nettoyage de rue, les rejets illicites au réseau pluvial (stationnement de camping car sur un parking) ou au milieu littoral (ports de plaisance).

Notons également les sources de pollution que peuvent représenter, par démultiplication, les systèmes d'assainissement autonome défectueux et, ce, aussi bien en temps sec qu'en temps de pluie.

\subsection{Quelques ordres de grandeur}

Les ordres de grandeur de la contamination bactériologique des rivières sont éminemment variables en fonction de la dilution des rejets de temps sec et de temps de pluie mais aussi de l'auto-épuration qui intervient dans la rivière pendant le transfert vers l'embouchure. C'est bien en termes de flux qu'il faut raisonner puisqu'un débit d' $1 \mathrm{~m}^{3} / \mathrm{s}$ et une concentration de $10^{3} \mathrm{E}$. Coli/ $100 \mathrm{ml}$ est équivalent au flux apporté à un débit de $10 \mathrm{~m}^{3} / \mathrm{s}$ et $10^{2} \mathrm{E}$. Coli/ $/ 100 \mathrm{ml}$. En revanche, lorsque l'on s'intéresse à des rejets du système d'assainissement situés à proximité d'une plage, on peut admettre les ordres de grandeurs suivants pour E. Coli $(\mathrm{n} / 100 \mathrm{ml})$ pour les hiérarchiser : - eaux usées brutes : $10^{7}$ à $10^{8}$,

- eaux usées après traitement biologique : $10^{5}$ à $10^{6}$, 
- eaux usées après traitement biologique et désinfection : quelques germes,

- eaux unitaires et/ou pluviales : de $10^{4}$ (séparativité totale) à $10^{6}$ (selon la dilution des eaux usées dans les eaux pluviales).

Ces ordres de grandeur appellent les remarques suivantes.

- Ils sont à comparer aux valeurs réglementaires :

- réglementation actuelle (article D1332-3 du code de la santé publique, transcrivant la directive 76/160/CEE du 8 décembre 1975) : valeur guide 100 et valeur impérative de 2000 ;

- réglementation future (zone côtière) : seuils de classement statistique à 250 et 500 (directive 2006/7/CE du 15 février 2006 qui doit être transcrite par les États membres au plus tard le 24 mars 2008).

- Même si le processus de mortalité des germes dans le milieu marin est important, ces quelques valeurs montrent que sur une plage fermée, présentant peu de renouvellement de l'eau, le moindre rejet d'eaux usées peut conduire à une dégradation marquée de l'eau de baignade et à l'existence d'un risque sanitaire pour les usagers de la baignade.

- Même si la concentration en germes d'une eau pluviale « stricte » est largement inférieure à celle des autres type d'eaux, les débits mis en jeu sont largement plus importants. Un rejet massif d'eaux pluviales pourra conduire à des rejets équivalents à quelques centaines voire quelques milliers de $\mathrm{m}^{3}$ d'eaux usées strictes (figure 1). Dans ce contexte, la configuration unitaire d'un réseau est de ce point de vue encore plus critique.

\subsection{Maîtriser ou éradiquer l'impact des rejets : quelles solutions ?}

Minimiser l'impact des rejets d'assainissement sur les eaux de baignade est un objectif pleinement présent dans les schémas directeur d'assainissement que mènent les collectivités du littoral, aux côtés d'enjeux plus traditionnels comme la lutte contre les inondations et la protection du milieu récepteur. De ces réflexions découlent la mise en place d'organes de régulation ou de stockage des effluents de temps de pluie avec comme objectif implicite de chercher à tout traiter et le mieux possible à l'exutoire du réseaux d'eaux usées, à savoir l'usine de dépollution des eaux usées.

Si cette stratégie est généralement admise pour la gestion des excédents de temps de pluie de faible ampleur dans les réseaux d'eaux usées, sur les réseaux unitaires, voire les réseaux d'eaux pluviales fortement souillés, outre la stratégie de «stockage restitution », d'autres solutions sont aujourd'hui opérationnelles. Par exemple, tolérer des déversements traités et désinfectés, en particulier lorsqu'ils ont un impact significatif sur les eaux de baignade, peut non

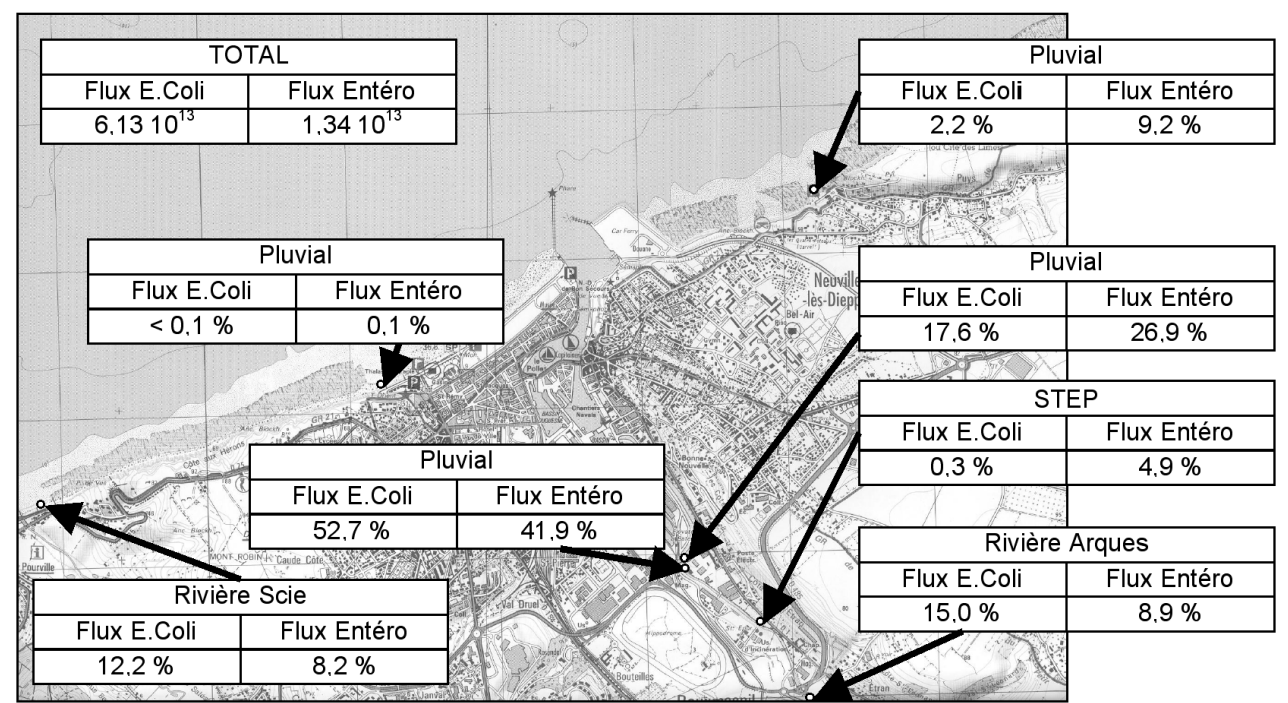

Figure 1. Bilan des flux de pollution rejetés en mer au droit de Dieppe pendant la pluie du 29 août 2003 (10 mm en 2 heures). Pour cette pluie, les flux de pollution bactériologique rejetée en mer sont équivalents à $200 \mathrm{~m}^{3}$ d'eaux usées brutes, ce qui représente un by-pass complet de la STEP pendant $1 \mathrm{~h} \mathbf{3 0}$ 
seulement améliorer le bilan des flux rejetés par le couple « réseau-usine » mais surtout minimiser les impacts globaux sur le milieu récepteur [TISSERAND, 2006].

Aux côtés de ces aménagements d'ordre structurel des systèmes d'assainissement, les aménagements fonctionnels ont également toute leur place. En premier lieu, assurer la séparation des réseaux permet à la fois de limiter les surcharges des réseaux d'eaux usées pendant les épisodes pluvieux mais également de limiter l'accumulation de la pollution par temps sec dans les collecteurs d'eaux pluviales; pollution immédiatement mobilisée et rejetée au milieu récepteur lors des pluies. En second lieu, il peut s'agir d'actions de réhabilitation des réseaux d'assainissement de façon à améliorer leur étanchéité et de limiter les infiltrations d'eaux parasites ainsi que les éventuelles exfiltrations vers le milieu récepteur.

Des pratiques préventives d'exploitation sont également extrêmement efficaces même si elles se limitent à des interventions ponctuelles avant la saison estivale : curages des collecteurs pluviaux, des « points noirs » des réseaux d'eaux usées ou de l'environnement immédiat des déversoirs d'orage.

Comme indiqué précédemment, les rivières côtières sont également les vecteurs de flux de pollution bactériologique, par temps sec comme par temps de pluie, qui échappent à la maîtrise d'ouvrage des collectivités du littoral. Sur certaines franges littorales où cohabitent tourisme et agriculture, elles peuvent être l'exutoire de la pollution bactériologique due aux élevages.

Agir sur les systèmes d'assainissement et sur l'élevage requiert alors une solidarité " amont-aval » entre l'arrière-pays et la frange littorale, particulièrement sensible aux retombées touristiques de la qualité des eaux de baignade.

De ce qui précède, le risque zéro de dégradation de la qualité des eaux de baignade peut malheureusement apparaitre comme un mythe :

- toutes les sources ne sont pas facilement contrôlables par des moyens conventionnels,

- les ouvrages contribuant à la maîtrise des rejets urbains par temps de pluie sont dimensionnés pour un niveau de protection donné (généralement pour une fréquence de retour de 3 à 12 mois).
Pour autant, faire de la bonne connaissance de l'impact potentiel des différents rejets un élément central des politiques d'aménagement peut permettre d'obtenir des impacts du système d'assainissement acceptables voire maîtrisables, notamment, dans le cadre des plans de gestion de la qualité des eaux de baignade prévus dans la nouvelle directive européenne.

\section{Importance de la courantologie côtière}

En zone marine côtière, l'impact des rejets d'assainissement va être modulé par deux facteurs prépondérants : d'une part, le transport et la dilution des effluents par les courants, d'autre part la capacité de survie des bactéries et des virus dans l'environnement marin. Sans négliger ce second point, nous allons cependant décrire avec plus de précision comment la connaissance de l'hydrodynamique côtière permet de comprendre l'essentiel de l'impact des rejets d'assainissement sur les plages et de hiérarchiser les programmes d'actions.

Par rapport à la causalité directe entre rejets amont et contaminations aval en rivière, la variabilité des courants marins introduit une complexité supplémentaire qu'on peut surmonter en se dotant de la capacité synoptique et prédictive qu'apporte la modélisation hydrodynamique numérique. On peut en effet se référer à des capteurs, mais s'ils sont in situ comme des courantomètres, l'aspect bi-dimensionnel, voire tridimensionnel, de la zone marine ne permet pas d'intégrer le régime de circulation comme c'est le cas de débitmètres bien positionnés sur des cours d'eau ; seul capteur synoptique actuel à l'échelle de la contamination côtière, restreinte à quelques kilomètres, le radar haute-fréquence mesure les courants de surface, mais il dispose d'une résolution spatiale tout juste suffisante, et ce dispositif, coûteux, n'est déployé que sur des sites sensibles du point de vue du trafic maritime. Ainsi, les modèles numériques, intégrant les équations de la physique, offrent-ils le seul moyen de suivi synoptique des courants. Afin d'observer les processus de transport et de diffusion sur des distances de quelques kilomètres, correspondant au déplacement des contaminations microbiologiques pendant leur survie en mer, la discrétisation de 
l'espace pour le calcul s'effectue sur des mailles de quelques dizaines de mètres. À l'intérieur des cellules ainsi définies, les paramètres physiques tels que courant, hauteur d'eau, température, salinité, sont considérés comme homogènes et les processus de taille inférieure sont représentés par un paramètre qui schématise cette turbulence, essentielle pour l'estimation de la diffusion verticale, mais surtout horizontale, des panaches. Pour déterminer l'emprise spatiale du modèle, en Manche et Atlantique, on vérifie que l'excursion de marée (trajet de particules conservatives durant un cycle flot-jusant) ne franchit pas les frontières du domaine de calcul.

Pour un modèle, la donnée statique de base, qui donne la configuration géométrique du domaine, est la bathymétrie, c'est-à-dire la topographie du fond de la mer. Exception faite des zones soumises à de larges transports sédimentaires (passes d'Arcachon, par exemple), cette donnée ne varie pas. Cependant, une acquisition à très haute résolution est nécessaire pour créer des modèles reproduisant fidèlement les découvrements en zone à fort marnage, les décollements de tourbillons et autres contre-courants près des caps, etc. Les zones de petits fonds n'ont pas fait l'objet de levés systématiques, et une campagne spécifique peut alors s'avérer nécessaire, par sondage acoustique (sondeurs multifaisceaux) ou télémétrie laser aéroportée (LIDAR).

Ensuite, par ordre d'influence décroissante, voici les principaux moteurs qui régissent l'hydrodynamique marine.

- Lamplitude de la marée, qui suit les éphémérides du soleil et de la lune. On dispose d'une base de données spatialisées de décomposition temporelle harmonique des hauteurs d'eau sur le pourtour du littoral (réf. SнOм), mais l'ajustement local des courants générés et du phasage exact des cycles reste une opération délicate. Dans la pratique, ce calage est opéré en faisant varier l'estimation du frottement des masses d'eau sur le fond, qui reflète la rugosité de la bathymétrie une fois encore à une échelle inférieure à celle de la maille de calcul.

- Les conditions atmosphériques, puisque vent et pression agissent par entraînement direct et par l'équilibre barométrique des hauteurs d'eau. Là encore, il est nécessaire de disposer de données météorologiques à une résolution compatible avec les processus en jeu dans le modèle. Des effets dus à l'orographie littorale, aux variations diurnes locales comme le régime des brises de chaleur, ne peuvent aujourd'hui être représentés que par des modèles atmosphériques fins emboîtés dans les prévisions générales, et dont la mise en œuvre n'est pas encore systématisée. Outre l'effet mécanique, les conditions atmosphériques influent sur les échanges thermodynamiques à l'interface air-mer (ensoleillement, conditions de nébulosité) et donc sur la part de circulation due à la convection thermique, les effets de stratification, etc.

- Les débits des fleuves, qui conditionnent la circulation haline. En effet, aux principales embouchures, les variations de densité dues à la salinité conditionnent des structures elles aussi stratifiées, et les apports d'eau massifs en période de crue modulent les courants en zone côtière.

- La circulation océanique globale, qui intervient comme condition aux limites du domaine côtier. Des tourbillons entrainnant des masses d'eau de plusieurs kilomètres de diamètre viennent déborder sur les plateaux continentaux. Leur position peut être déterminée grâce aux mesures satellitaires d'altimétrie, et leur prévision fait l'objet désormais de bulletins fournis par l'océanographie opérationnelle.

- Létat de mer, c'est-à-dire la formation de vagues à plusieurs échelles, se déduit aussi des conditions météorologiques, avec une notion d'intégration temporelle et spatiale très importante pour ce qui est de la formation de houle. Linterface mer-air s'observe (autre usage des radars haute fréquence et possibilité de télédétection spatiale) et se modélise, avec par conséquent une capacité de prévision ; dans une première approche, cette rugosité interfère dans l'efficacité d'entraînement du vent, dans une participation mineure à la circulation par fonds moyens et plus accentuée dans la zone de déferlement. Par ailleurs, la survie des organismes microbiologiques étant allongée par la turbidité des eaux (protection visà-vis des rayons UV, attachement à la matière en suspension), la remise en suspension des sédiments par les vagues permet de raffiner les simulations. Un modèle de courantologie côtière prend par conséquent tout ou partie de ces paramètres en compte, 
suivant le degré de précision requis pour l'application. Les particularités de la zone d'intérêt permettent aussi des simplifications : si les phénomènes de stratification sont rares, dans un domaine soumis à de forts courants de marée en particulier, la modélisation peut se limiter à une circulation bidimensionnelle, négligeant ainsi les différences pouvant intervenir dans la colonne d'eau.

Il faut aussi distinguer si l'usage du modèle vise une étude d'impact ou bien une prévision opérationnelle. Dans le premier cas, ce sont les scénarios les plus probables combinant ces divers facteurs qui sont simulés : les bureaux d'études déterminent ainsi le positionnement optimal d'un émissaire dans un fort courant divergent, l'effet d'un diffuseur, etc. La prévision opérationnelle permet quant à elle de produire des alertes sur les zones sensibles telles que les plages, en fonction de l'étendue calculée des panaches : dans le cas du SAERS développé sur Dieppe (cf. paragraphe 4), ceci conduit à une intensification des prélèvements avant la prise de décision. Une étape ultérieure, plus complexe, pourrait consister en une gestion interactive des horaires de rejet, de manière à minimiser l'impact : rétention en période de fortes précipitations, cadencement des largages sur le cycle de marée modulé par les conditions météorologiques.
Le risque varie ainsi au sein du cycle de marée et de façon spécifique à chaque plage (selon la position de la plage par rapport aux sources de pollution et le jeu des courants locaux). On peut cependant dans une première approche construire un système opérationnel à partir d'une base de scénarios, combinant des valeurs fixes des paramètres dont l'action est prépondérante sur l'intensité et l'extension des panaches. C'est ce que propose le système SAERS (cf. paragraphe 4).

De l'expérience réalisée sur les plages du pays de Caux, voici quelques enseignements qui démontrent l'importance d'une bonne estimation de la courantologie côtière.

- Le volume et la charge d'un rejet ne déterminent pas son impact : les émissaires d'eaux pluviales de Dieppe se jettent dans le port, qui joue un rôle de lagunage, surtout en mortes eaux, car l'excursion de marée sort peu du port. Qui plus est, la jetée du port de Dieppe rejette au large le panache de l'Arques à marée descendante. Par contre, la plage de Dieppe peut être vulnérable aux rejets de la Scie, dont l'embouchure est située à $3 \mathrm{~km}$ à l'ouest et moins chargée en flux que les émissaires d'eaux pluviales de Dieppe. Cette vulnérabilité se manifeste surtout dans certains

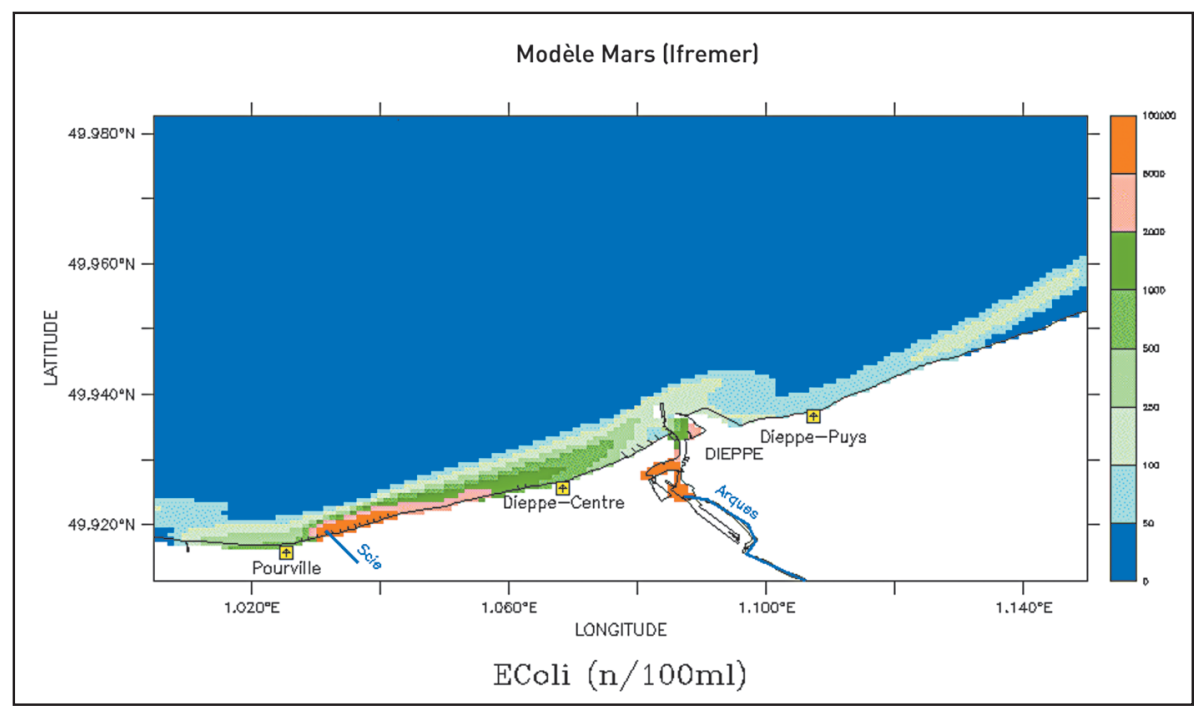

Figure 2. Simulation de la dispersion des apports microbiologiques sur les plages du Pays de Caux, dans le cadre du SAERS. Le modèle, à maille de $75 \mathrm{~m}$, permet de représenter la variabilité de l'extension et de la direction des panaches sur une période de quelques heures, en fonction de paramètres prédominants tels que la marée, la direction et l'intensité du vent, la charge des rejets. La prévision de dépassement de seuil sur les plages cibles déclenche un prélèvement de contrôle à lecture rapide, aide à la décision pour la gestion de l'ouverture des plages 
contextes très particuliers (Scie chargée suite à un épisode pluvieux, mortes eaux, vent de nord-ouest).

- La morphologie du lieu doit être précisément prise en compte : au-delà de la précision de la bathymétrie, on peut rendre compte de dispositifs assez complexes mais déterminants, comme le rôle de déflecteur de la digue ajourée qui finalement, au jusant, protège la grande plage de Dieppe des rejets de l'Arques.

- Dans une zone à marée, les impacts suivent un horaire précis, que les vents dominants peuvent moduler. On peut donc distinguer des impacts immédiats et des impacts différés. À Dieppe, la plage de Puys est clairement située sous l'influence de deux sources de pollution. La première est constituée du rejet d'un collecteur pluvial et son impact sur la plage peut être quasi immédiat. La seconde source de pollution est le panache de l'Arques qui peut se rabattre au droit de la plage aux alentours de la pleine mer et ce, dans les 48 heures qui suivent un épisode pluvieux majeur (impact différé). Ainsi, sur cette plage, dans $15 \%$ des situations de rejet par temps de pluie, un impact différé dans le temps est possible.

Pour affiner un tel système, on peut être tenté de discrétiser finement les paramètres mentionnés ci-dessus. Malheureusement, la combinatoire de facteurs devient rapidement incommensurable. En ce cas, il faut avoir recours à une modélisation quotidienne.
La mise en place progressive de l'océanographie opérationnelle côtière prépare la production de tels résultats, sur lesquels on peut brancher des systèmes d'alerte. Ceci exige cependant la permanence d'une expertise pour qualifier les résultats de prévision.

\section{Vers une maîtrise opérationnelle du risque}

La dernière partie de cet article est développée plus complètement dans la communication ${ }^{5}$ « Modélisation et scénarios d'alertes : exemple de Dieppe. » du congrès Astee 2006.

Dieppe constitue un site pilote de la démarche de gestion active et ce, depuis l'été 2003. L'amélioration et la maîtrise de la qualité des eaux de baignade est une préoccupation majeure historique de la collectivité qui structure sa politique d'amélioration de la performance du système d'assainissement.

Sur ce site, la première particularité de la démarche de « maîtrise opérationnelle des risques sanitaires » résulte de l'importance donnée à la surveillance effective des sources de pollution qui, en période de temps sec et hors incidents, permet de limiter le volet analytique à quelques analyses de routine par semaine par la méthode rapide Coliplage ${ }^{\circledR}$. Ces analyses de routine ont pour objet de détecter

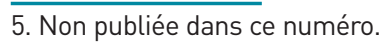

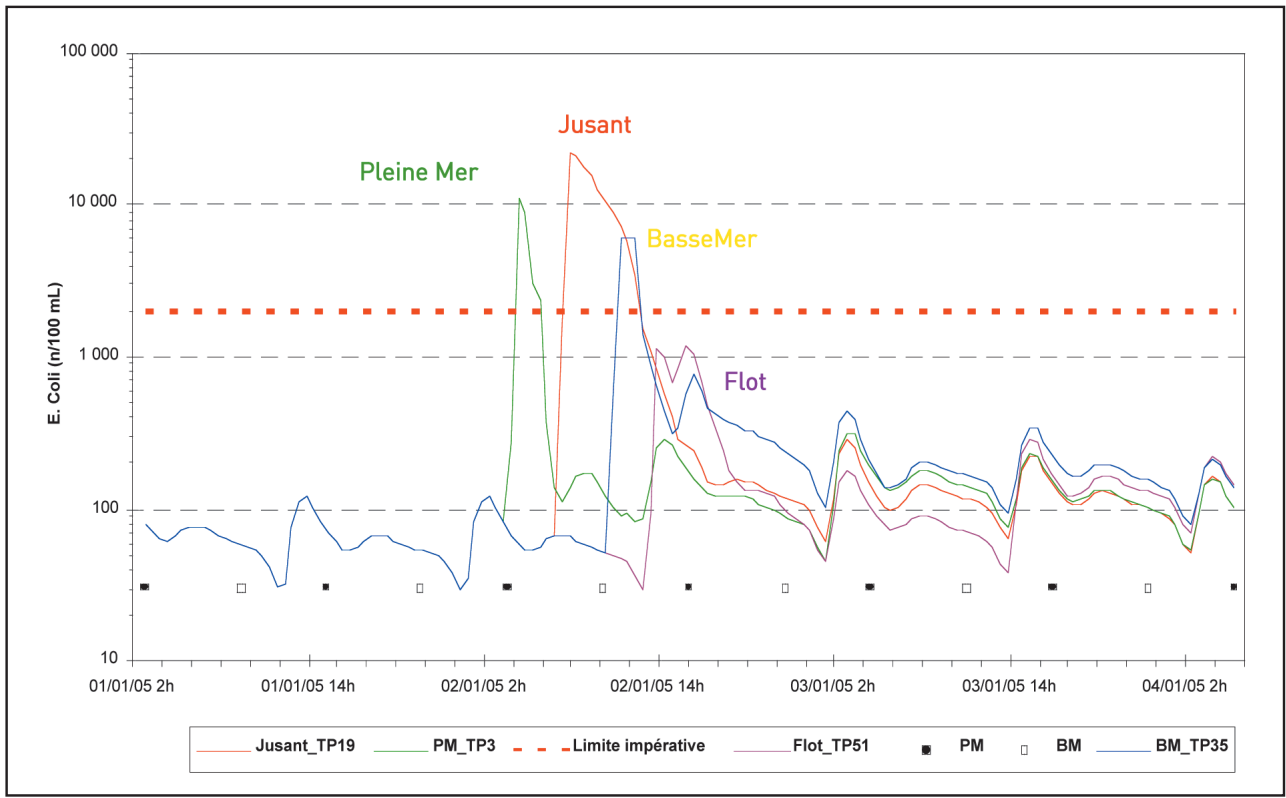

Figure 3. Étude de sensibilité vis-à-vis de la synchronisation de la pluie avec le cycle de marée. Le déphasage en temps s'accompagne d'une modulation de l'amplitude et de la durée du pic de contamination 
d'éventuelles pollutions en provenance de l'amont des bassins-versants et qui seraient véhiculées par les rivières côtières (en l'occurrence l'Arques et la Scie). De telles pollutions échapperaient alors au dispositif de surveillance de la totalité des ouvrages d'assainissement de la frange littorale.

La seconde caractéristique de la gestion active sur Dieppe provient d'une évaluation quotidienne des risques qui conditionne la réalisation ou non d'analyses complémentaires à celles prévues en routine (toujours avec la méthode Coliplage ${ }^{\circledast}$ ). Cette évaluation quotidienne des risques intègre les données d'autosurveillance des ouvrages d'assainissement (historique des dernières 36 heures) et les résultats d'un modèle courantologique côtier. Ce modèle est le fruit du partenariat entre Veolia et l'Ifremer. Au-delà du modèle lui-même, c'est davantage sa déclinaison opérationnelle, pour un usage quotidien par des utilisateurs non spécialistes, qui marque la véritable originalité de ce partenariat.

Pour concevoir le « Système d'aide à l'évaluation des risques sanitaires » (SAERS), une étude de sensibilité préalable a été entreprise : sont ainsi pris en compte deux coefficients de marée, plusieurs directions et intensités de vent représentatives des régimes météorologiques les plus courants et les plus « impactants » (certaines directions de vent ont une influence négligeable sur la circulation). Ces combinaisons sont augmentées de facteurs liés à l'hydrologie terrestre, non pas qu'ils affectent la circulation hydrodynamique, mais parce qu'ils déterminent l'intensité des flux de contamination (pluie, antécédents pluvieux). C'est ainsi une centaine de scénarios de référence qui ont été pris en compte par les équipes de Veolia et de l'Ifremer. Validée par une analyse de sensibilité et par un suivi rigoureux de la pertinence des prévisions introduites dans la base de données, cette méthode possède l'avantage de reposer sur des modélisations préalables.

En cours d'exploitation, l'opérateur n'a besoin que de sélectionner les valeurs discrètes des paramètres se rapprochant le plus des cas pré-simulés ; il est aidé en cela par une interface, qui en sélectionne certains de façon automatique, tels que le coefficient de marée le plus représentatif en fonction de la date. Il a alors accès à des résultats lui permettant d'évaluer les risques de dégradation de la qualité des eaux de baignade au cours des prochaines heures (figures $4 a$ et $4 b$ ).

Le SAERS a été utilisé en routine pendant deux mois pour les deux plages de Dieppe durant l'été 2005. Il permet d'anticiper jusqu'à un horizon de 24 heures les risques de pollution à venir au droit des deux

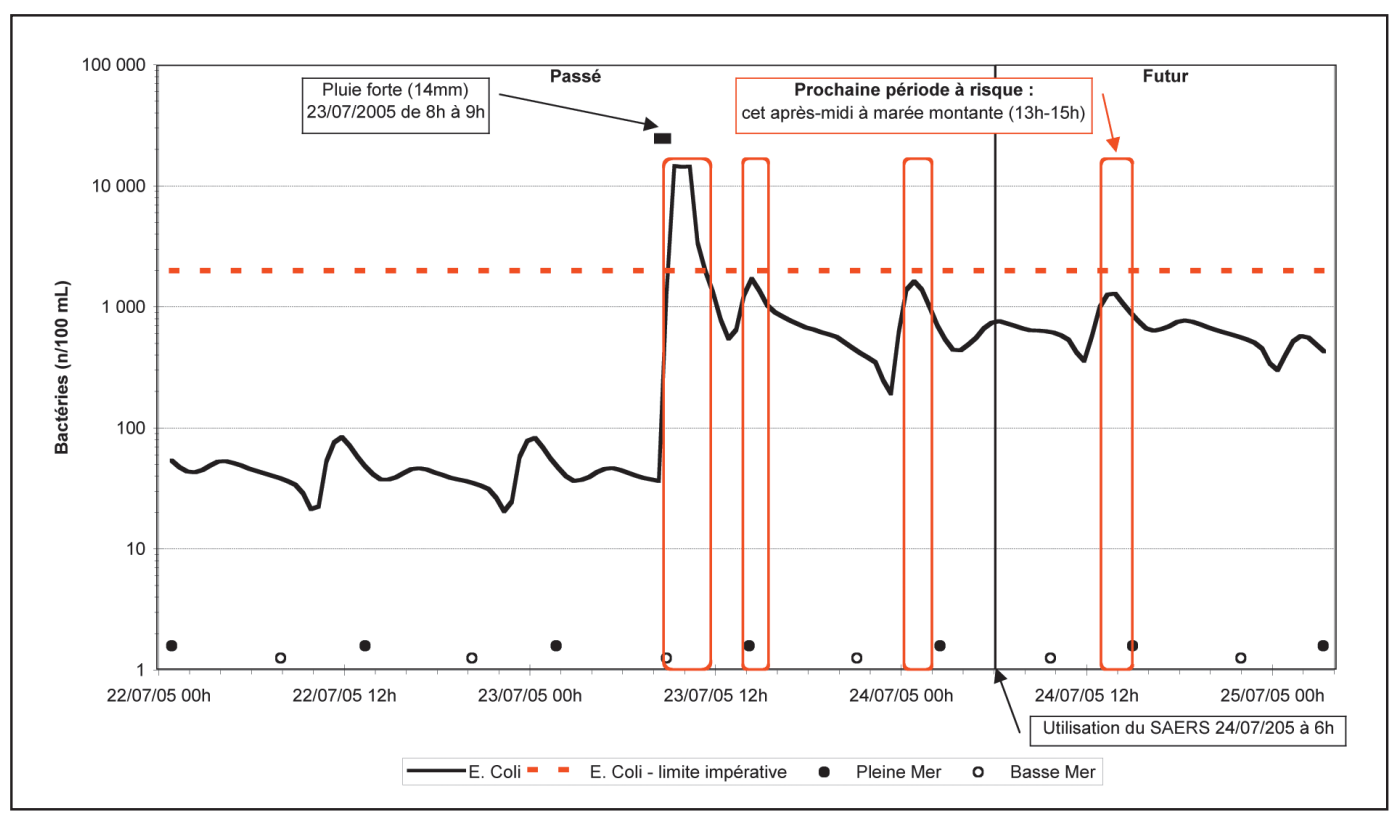

Figure 4a. Prévision de pollutogramme de la plage de Puys. Les prélèvements complémentaires réalisés lors d'une estimation de dépassement de seuil (ici, seuil réglementaire actuel de $2000 \mathrm{E}$. Coli/100 $\mathrm{ml}$ ) sont prescrits en fonction de l'heure à laquelle le pic se produit 


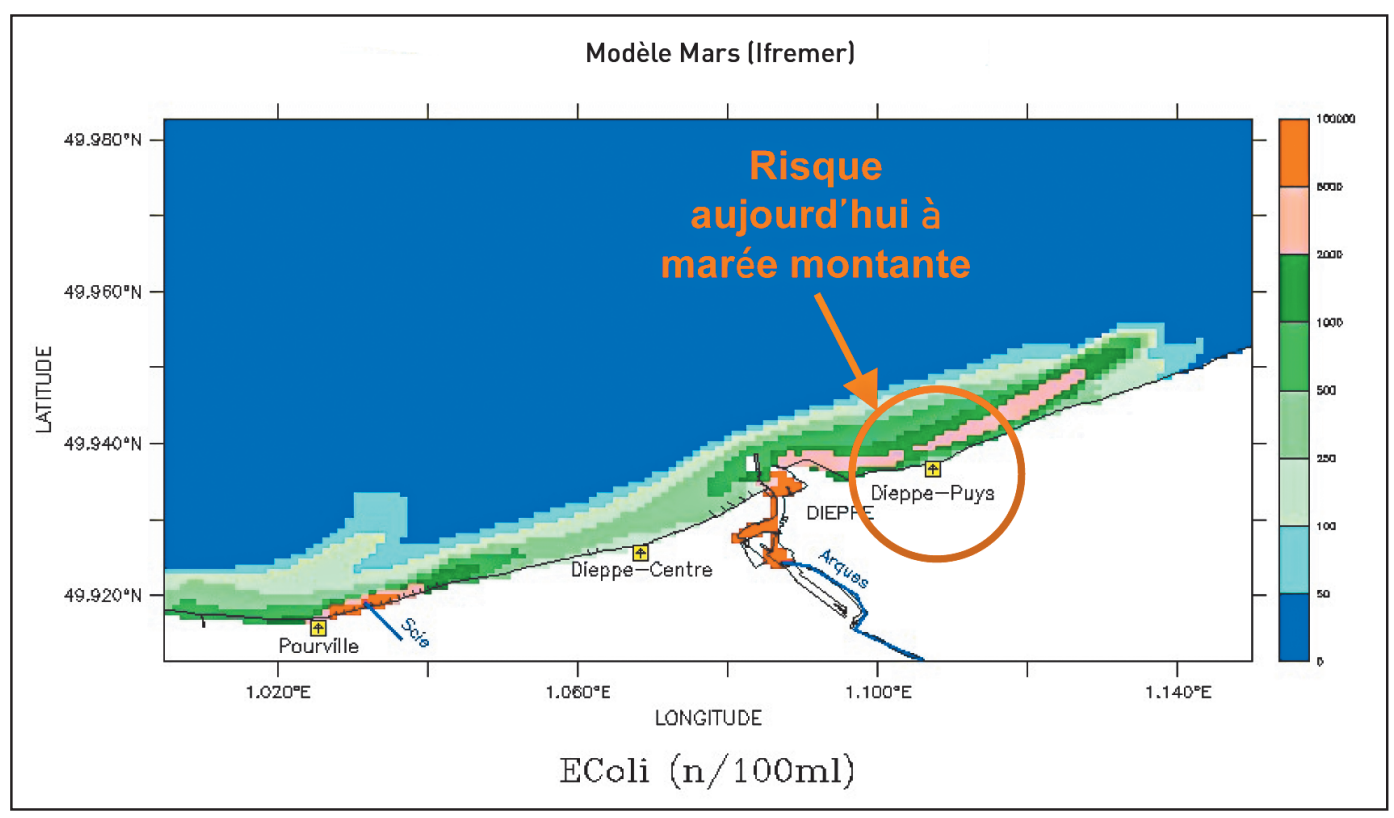

Figure 4b. Prévision de dispersion des apports microbiologiques sur les plages du Pays de Caux associée à la situation de la figure $4 a$

plages de Dieppe, et, ce, compte tenu de l'historique des 36 dernières heures.

Dans un contexte de courants de marée très marqués, comme c'est le cas au droit du Pays de Caux, il a pu être ainsi validé que l'usage d'un modèle courantologique côtier permet d'introduire une véritable dimension prédictive à la gestion active.

Couplé à la méthode d'analyse rapide de l'eau de baignade Coliplage $^{\circledast}$, à une organisation et une ergonomie adéquates, ce modèle permet la production d'informations objectives, pleinement utiles à la prise de décision des élus, notamment en cas de risque avéré de dégradation de la qualité des eaux de baignade.

En outre, à travers son extension à 100 scénarios, il permet de disposer des éléments de connaissance sur les sources de pollution qui ont une influence prépondérante sur la qualité des eaux de baignade au droit des plages, base de tout diagnostic hiérarchisé précédant une démarche d'éradication progressive des sources de pollution identifiées.

\section{Conclusion}

La nouvelle directive des eaux de baignade modifiera les règles de classement des plages dont il conviendra de regarder les conséquences au cas par cas (plage par plage). Cette projection a déjà été initiée par les agences de l'eau. Limpact de ces nouvelles règles concernera majoritairement une partie des plages aujourd'hui régulièrement classées en C et en B. Pour ces plages, des programmes d'éradication des sources de pollution chroniques seront sans doute nécessaires, ce qui impliquera un effort accru, très souvent déjà anticipé dans les documents de programmation que constituent les SAGE et les SDAGE, notamment dans la mise en cuvre d'ouvrages d'assainissement performants et fiables en zone littorale ou proche littorale.

En revanche, indépendamment de l'importance des efforts qui pourront être consentis, il conviendra sans doute de faire la part entre ce qui relève des pollutions chroniques, qui se manifestent en temps sec, et ce qui relève de la pollution événementielle, incident et surtout pluviométrie. En effet, l'éradication des sources de pollution chronique en provenance des systèmes d'assainissement renvoient à des solutions techniques aujourd'hui connues et globalement bien maîtrisées. Pour les dégradations de la qualité des eaux de baignade suite à des pluies, il est à craindre qu'une proportion des plages (dont certaines aujourd'hui classées en A) resteront malheureusement toujours vulnérables à ce type d'événements. C'est davantage les plans de gestion qui permettront de limiter les conséquences sanitaires de ces dégradations temporaires pour les usagers de la plage. 
Dans ce contexte, la courantologie côtière pourra permettre d'optimiser les investissements en phase de programmation des ouvrages conformément à un usage aujourd'hui dûment admis et répandu dans les schémas directeurs d'assainissement. De plus, elle pourra être partie prenante des plans de gestion des risques par une discrimination objec- tive des facteurs conditionnant ou non l'impact des rejets au droit des plages. Ce nouvel usage, testé en routine et avec succès à Dieppe durant l'été 2005, permet, dans un contexte marqué par les courants de marée, de conférer une véritable dimension prédictive à la « gestion active de la qualité des eaux de baignade ».

\section{Bibliographie}

BECHMANN G., 1899 : "Salubrité Urbaine - Distributions d'eau et assainissement". Encyclopédie des Travaux Publics

GOUBERT J.-P., 1986 : "La conquête de l'eau". Ed. Robert Laffond.

GUILLAUD J.F., DERRIEN A., GOURMELON M., 1997. " T90 as a tool for engineers : interest and limits. "Wat. Sci. Tech. 35, 277-281.

POMMEPUY M., DUMAS F., CAPRAIS M.P., CAMUS P. : "Sewage impact on shellfish microbial contamination". Water Science Technology. Vol 50, pp 117-124, 2004. POMMEPUY M., SALOMON, J.C., 1991 : "A mathematical model for enteric bacteria in coastal areas". In Environmental Lee \& Cheung Ed Hydraulics. Balkema, Rotterdam, 841-846.
RIOU Ph., DUMAS F., BLANCHET F., 2006 : "Modélisation et scénarios d'alertes : exemple de Dieppe". Congrès Astee, Deauville.

SALOMON J.C., POMMEPUY M., 1990 : "Mathematical model of bacterial contamination of the Morlaix estuary (France)". Water Research, 24, 983-994.

TISSERAND B., FORGEREAU P., BLANCHET F., 2005 : "Assainissement et qualité des eaux de baignade: retours d'expériences après deux saisons de surveillance active sur différentes zones littorales". Congrès Astee, Paris.

TISSERAND B. 2006 : "Eaux pluviales : efficacité d'un traitement combiné à une désinfection aux UV". Congrès Astee, Deauville.

\section{Résumé}

\section{F. BLANCHET, E. SOYEUX, J.-C. DEUTSCH}

Y.H. DE ROECK. Impact des rejets

d'assainissement permanents ou transitoires sur la qualité des eaux de baignade

La vulnérabilité des eaux de baignade aux rejets d'assainissement constitue à ce jour une sorte de « fait acquis ». Grâce aux progrès accomplis durant les quinze dernières années, les rejets de temps sec présentent aujourd'hui un caractère accidentel. La vulnérabilité aux rejets par temps de pluie, souvent massifs du fait du nombre de points de déversement et de leur extension géographique, est certes connue des « spécialistes » mais en général encore mal appréhendée. La nouvelle directive européenne sur les eaux de baignade introduit tout un ensemble de dispositions qui visent une meilleure maîtrise des sources de pollution : sur le long terme, plan d'éradication ou de diminution en cas d'insuffisance et sur le court terme, plan de gestion supposant un niveau de surveillance adéquat de ces rejets. Ces dispositions, cohérentes avec celles de la directive cadre sur l'eau (DCE), auront des répercussions techniques importantes que cette communication se propose de décrire, comme notamment, l'influence de la courantologie côtière, la pollution bactériologique des eaux pluviales Imême en zone séparative) et les solutions d'amélioration envisagées pour limiter l'importance des impacts des rejets d'assainissement. 